\title{
Molecular profiling of signalling proteins for effects induced by the anti-cancer compound GSAO with $\mathbf{4 0 0}$ antibodies Verity A Cadd ${ }^{1}$, Philip J Hogg ${ }^{2}$, Adrian L Harris ${ }^{3}$ and Stephan M Feller*1
}

Address: ${ }^{1}$ Cancer Research UK Cell Signalling Group, Weatherall Institute of Molecular Medicine, University of Oxford, UK, ${ }^{2}$ Centre for Vascular Research, University of New South Wales, Sydney 2052 and Children's Cancer Institute Australia for Medical Research, Randwick 2031, Australia and ${ }^{3}$ Cancer Research UK Growth Factor Group, Weatherall Institute of Molecular Medicine, University of Oxford, UK

Email: Verity A Cadd - verity.cadd@cancer.org.uk; Philip J Hogg - p.hogg@unsw.edu.au; Adrian L Harris - aharris.clin@cancer.org.uk; Stephan M Feller* - stephan.feller@cancer.org.uk

* Corresponding author

Published: 09 June 2006

BMC Cancer 2006, 6:155 doi:10.1186/147|-2407-6-155
Received: 06 February 2006

Accepted: 09 June 2006

This article is available from: http://www.biomedcentral.com/I47I-2407/6/155

(c) 2006 Cadd et al; licensee BioMed Central Ltd.

This is an Open Access article distributed under the terms of the Creative Commons Attribution License (http://creativecommons.org/licenses/by/2.0), which permits unrestricted use, distribution, and reproduction in any medium, provided the original work is properly cited.

\begin{abstract}
Background: GSAO (4-[N-[S-glutathionylacetyl]amino] phenylarsenoxide) is a hydrophilic derivative of the protein tyrosine phosphatase inhibitor phenylarsine oxide (PAO). It inhibits angiogenesis and tumour growth in mouse models and may be evaluated in a phase I clinical trial in the near future. Initial experiments have implicated GSAO in perturbing mitochondrial function. Other molecular effects of GSAO in human cells, for example on the phosphorylation of proteins, are still largely unknown.
\end{abstract}

Methods: Peripheral white blood cells (PWBC) from healthy volunteers were isolated and used to profile effects of GSAO vs. a control compound, GSCA. Changes in site-specific phosphorylations, other protein modifications and expression levels of many signalling proteins were analysed using more than 400 different antibodies in Western blots.

Results: PWBC were initially cultured in low serum conditions, with the aim to reduce basal protein phosphorylation and to increase detection sensitivity. Under these conditions pleiotropic intracellular signalling protein changes were induced by GSAO. Subsequently, PWBC were cultured in 100\% donor serum to reflect more closely in vivo conditions. This eliminated detectable GSAO effects on most, but not all signalling proteins analysed. Activation of the MAP kinase Erk2 was still observed and the paxillin homologue Hic-5 still displayed a major shift in protein mobility upon GSAO-treatment. A GSAO induced change in Hic-5 mobility was also found in endothelial cells, which are thought to be the primary target of GSAO in vivo.

Conclusion: Serum conditions greatly influence the molecular activity profile of GSAO in vitro. Low serum culture, which is typically used in experiments analysing protein phosphorylation, is not suitable to study GSAO activity in cells. The signalling proteins affected by GSAO under high serum conditions are candidate surrogate markers for GSAO bioactivity in vivo and can be analysed in future clinical trials. GSAO effects on Hic-5 in endothelial cells may point to a new intracellular GSAO target. 


\section{Background}

The term 'cancer' encompasses a wide variety of distinct, multigenic diseases. Even within a specific tumour type, a remarkable degree of heterogeneity on the level of DNA lesions and affected signalling pathways is apparent. Many cancer relevant signalling molecules, but also many molecular targets of anti-cancer drugs, therefore remain unknown. Prominent examples of signalling protein classes long known to be involved in generating cancer pathologies include GTPases, protein kinases and transcription factors. By contrast, protein phosphatases have only recently entered the stage as known players in cancer development. At least 30 protein phosphatases are now implicated in cancer development and other human diseases [1-3]. In some of these cases, mutational inactivation of a protein phosphatase appears to mimic the constitutive activation of its target kinase(s) [3]. In other cases, hyperactivation or deregulation of a phosphatase may contribute to kinase activation. For example, overexpression of the Cdc 25 family phosphatases, which control cell cycle progression, is well documented for a variety of cancers, making the Cdc 25 proteins interesting potential targets for anti-cancer therapies [4-7] and references therein).

The modulation of specific cellular signalling pathways to treat human cancers has only recently developed into an area of intense clinical research activity. A large number of clinical trials for novel signal transduction modulator (STM) drugs are currently planned or under way. STM drugs often have relatively low toxicity, so determination of the maximum tolerated dose (MTD) may not be a prime goal for phase I clinical trials. Instead, identification of an optimal biologically active dose (OBD) is essential [8]. Rapid determination of the OBD requires that in vivo markers of drug activity are available before or very early during the clinical trial.

This study identifies several proteins in PWBC which are affected by the novel anti-cancer compound GSAO (4-[N[S-glutathionylacetyl]amino] phenylarsenoxide) [9] (Figure 1A). They may be useful as clinical surrogate markers to monitor or predict the anti-cancer activity of GSAO and could also help to provide further insight into the biological mechanisms of GSAO action.

GSAO has anti-angiogenic activity in vitro and in vivo [10]. Mitochondria and in particular the adenine nucleotide translocator (ANT) have been described as one target of GSAO. However, mitochondria are present in virtually all living cells. Therefore, inhibition of ANT does not per se explain the low toxicity and anti-angiogenic activity of GSAO.

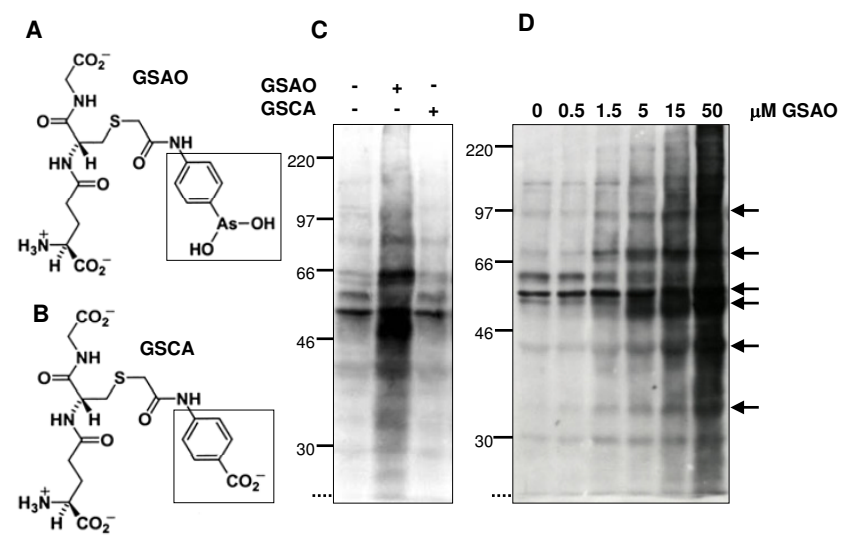

Figure I

Chemical structure GSAO and GSCA and Dosedependent increase of PWBC protein tyrosine phosphorylation induced by GSAO. A and B. The boxes indicate the region of the molecule thought to be primarily responsible for the biological activity of GSAO and the corresponding region in the control GSCA. Oxidation of GSAO is possible. The resulting molecule (GSAA) is thought to be inactive, but may become active again, if reduced in vivo. To avoid oxidation, GSAO solutions are prepared freshly, or stored after snap-freezing at $-80^{\circ} \mathrm{C}$. No significant activity loss of deep-frozen GSAO was observed for up to 6 months. PWBC were treated with GSAO or GSCA where indicated for $24 \mathrm{~h}$ and tyrosine phosphorylation detected by western blot. C $50 \mu \mathrm{M}$ GSCA does not alter the protein PTyr levels of PBWC detectably. D Changes in protein pTyr patterns are apparent with as little as I.5 $\mu \mathrm{M}$ GSAO. GSAO-affected proteins are indicated with arrows.

Recent studies have revealed that differences in multidrug resistance proteins (MRP1 and 2) and cellular glutathione levels [11] appear to contribute to the preferential in vivo activity of GSAO in endothelial cells. Nevertheless, it is well known that practically all drugs in clinical use have multiple molecular in vivo targets. They depend on the affected cell type and applied doses. Some of these targets are intimately linked to the adverse effects of the drug. In cancer cells, with their heterogeneous genetic lesions, drug targets can even be present in different combinations or at different expression and activity levels when comparing individual patients. Identification and monitoring of novel molecular targets could thus help to understand why a drug is more effective or toxic in some patients compared to others. PWBC from donated blood were chosen for the analyses, since they are easily obtainable from healthy volunteers prior to clinical trial and later from the patients enrolled in the trials. A main focus of the experiments was to detect changes in protein phosphorylation induced by GSAO treatment, because GSAO is a hydrophilic derivative of phenylarsine oxide (PAO), a well known protein phosphatase inhibitor $[12,13]$. In contrast to GSAO, PAO is unsuitable for in vivo applica- 
tions and toxic for cells at nanomolar concentrations [10]. It penetrates very rapidly into cell membranes and is hence not equally distributed throughout the vascular system of an organism. As GSAO is expected to enter a phase I clinical trial in the near future a molecular profiling of its effects on signalling proteins prior to the trial was performed. The aim was to provide data for trial-supporting pharmacodynamic molecular assays, trial design and possibly identification of additional mechanisms of GSAO action.

\section{Methods \\ Isolation and culture of PWBC from healthy human volunteers}

PWBC were obtained from 14 healthy volunteers not taking any medication ( 8 male, 6 female; aged between 23 and 60 years) with informed consent. Ethical approval was granted by the Oxfordshire Ethics Committee (study number 05/Q1605/59). Cell preparations from more than 40 different blood donations were used. All blood donations took place between 9 and 11 a.m. to minimise potential circadian changes of signalling proteins. Whole blood $(54 \mathrm{ml})$ was collected from a peripheral arm vein into $6 \mathrm{ml}$ of $46.7 \%(\mathrm{v} / \mathrm{v})$ trisodium citrate (final concentration $4.67 \%$ ) and syringes immediately placed on a nutator at room temperature for at least $5 \mathrm{~min}$ to ensure complete mixing. Batches of $20 \mathrm{ml}$ citrated blood were then carefully layered onto $20 \mathrm{ml}$ Polymorphprep ${ }^{\mathrm{TM}}$ density gradient media (Axis Shield PoC AS, Oslo, Norway) and centrifuged at $500 \times \mathrm{g}$ for $30 \mathrm{~min}$ at $20^{\circ} \mathrm{C}$ to remove erythrocytes. PWBC were collected from the medium/ serum interface, transferred into a $50 \mathrm{ml}$ centrifuge tube and gently resuspended in sterile PBS. PWBC were then centrifuged for $20 \mathrm{~min}$ at $600 \times \mathrm{g}$ at $20^{\circ} \mathrm{C}$, PBS was aspirated and the PWBC pellet stored in ice. PWBC were subsequently cultured in either $100 \%$ donor serum or RPMI1640 supplemented with $0.5 \%$ foetal bovine serum (FBS), $100 \mu \mathrm{g} / \mathrm{ml}$ penicillin-streptomycin and $2 \mathrm{mM} \mathrm{L-}$ glutamate. Donor serum was simultaneously prepared freshly from peripheral blood. For this, blood was collected without anticoagulant and centrifuged for $30 \mathrm{~min}$ at $3700 \times \mathrm{g}$ at $20^{\circ} \mathrm{C}$. The supernatant was transferred into a fresh centrifuge tube and incubated for $2 \mathrm{~h}$ at room temperature with nutation to allow coagulation, before centrifugation at $3700 \times \mathrm{g}$ for $30 \mathrm{~min}$ at $20^{\circ} \mathrm{C}$ to obtain serum. Pelleted PWBC were resuspended in either growth medium or donor serum and equilibrated for $2 \mathrm{~h}$ at $37^{\circ} \mathrm{C}$ in a humidified cell culture incubator with $5 \% \mathrm{CO}_{2}$, before treatment with the compound of interest. Cells were treated with either GSAO, the active drug, or 4-N-(Sglutathionyl acetyl) amino) benzoic acid (GSCA) in which the trivalent arsenic of GSAO has been replaced by a carboxylic acid, as a control, for the concentrations and durations indicated. Stock solutions of both GSCA and GSAO were made at a concentration of $3 \mathrm{mM}$ in RPMI
1640 and, since GSAO is prone to oxidation, stored in aliquots at $-80^{\circ} \mathrm{C}$ unless used immediately. PAO was freshly dissolved in cell culture grade DMSO at a concentration of $10 \mathrm{mM}$ and diluted to $1 \mu \mathrm{M}$ with medium.

\section{Preparation and analyses of protein lysates}

Treated or mock-incubated PWBC were scraped from tissue culture flasks, transferred to a $50 \mathrm{ml}$ centrifuge tube filled with PBS and centrifuged at $3700 \times \mathrm{g}$ for $20 \mathrm{~min}$ at $20^{\circ} \mathrm{C}$ to pellet the cells. The PBS was completely aspirated from the PWBC pellet and the pellet lysed directly in boiling SDS-PAGE sample buffer (70 mM TrisHCl pH6.8, 5\% (v/v) $\beta$-mercaptoethanol, 40\% (v/v) glycerol, 3\%(w/v) SDS, $0.05 \%(\mathrm{w} / \mathrm{v})$ bromophenol blue). The samples were then boiled for a further $5 \mathrm{~min}$, cooled to room temperature and sonicated for 30 seconds to fragment DNA contained in the cell lysate. Proteins in the lysate were resolved by SDS polyacrylamide gel electrophoresis (SDSPAGE) and electrotransferred to PVDF membranes (Hybond P, Amersham Biosciences). The membranes were blocked and probed overnight at $4{ }^{\circ} \mathrm{C}$ with different phospho-epitope specific or other antibodies according to the antibody manufacturers instructions. Bound primary antibodies were detected using horseradish peroxidaseconjugated anti-mouse or anti-rabbit immunoglobulins (Jackson ImmunoResearch Inc.) and ECL (Amersham Biosciences). Equal loading of different protein extract samples was routinely confirmed by Coomassie-blue staining of an SDS-PAGE-separated aliquot prior to blot analyses. Antibodies used in this study are compiled in the additional files 8 and 9. The BD PowerBlot Western Array Screen Service (BD Biosciences) was utilised to analyse expression levels of 240 individually selected signalling proteins using monoclonal antibodies.

\section{Culture and treatment of HUVEC}

Human umbilical vein endothelial cells (HUVEC) were purchased from TCS Cell Works, (Botolph Claydon, UK) and cultured according to the manufacturers conditions with recommended growth media, cell attachment factor and passage conditions. Cells were cultured in $10 \mathrm{~cm}$ plates until $80 \%$ confluent before treatment with GSAO or GSCA in growth media with high serum (15\% FBS) for 24 hours at $37^{\circ} \mathrm{C}$ with $5 \% \mathrm{CO}_{2}$. Cells were lysed with boiling sample buffer. Protein analysis, SDS PAGE and Western blots were carried out as described for the PWBC.

\section{Results \\ Pleiotropic effects of GSAO on PWBC cultured in low serum conditions}

In initial experiments it was determined how rapidly protein phosphorylation changes were detectable upon GSAO treatment of freshly isolated PWBC from healthy donors under low serum culture conditions. For this, PWBC were incubated with GSAO or its more hydropho- 
bic relative PAO. As previously reported in the literature for various cell types, increased tyrosine phosphorylation was rapidly observed with PAO. Incubation of PWBC with $1 \mu \mathrm{M}$ PAO for $1 \mathrm{~h}$ resulted in a hyper-phosphorylation of protein-tyrosyl residues that was comparable to an incubation of PWBC with $50 \mu \mathrm{M}$ GSAO for $8 \mathrm{~h}$ (additional file 1 ). This was not unexpected, since GSAO is specifically modified to greatly slow down its uptake into cells. Incubation of PWBC with a single dose of GSAO for $24 \mathrm{~h}$ instead of $8 \mathrm{~h}$ greatly enhanced the hyperphosphorylation of proteins on tyrosine residues (figure 1C). Treatment with $50 \mu \mathrm{M}$ of the control compound GSCA (see figure 1B for structure), which lacks the crucial arsenic moiety, did not detectably affect basal PWBC protein-tyrosyl phosphorylation (figure 1C). Subsequently, titration experiments showed that incubation of PWBC for $24 \mathrm{~h}$ with 1.5 $\mu \mathrm{M}$ GSAO readily induced hyper-phosphorylation (figure 1D). Similar results were obtained for threonine phosphorylation of proteins upon GSAO treatment of PWBC (additional file 2). Nine different commercial anti-pSer antibodies were also tested, but all were found not to be suitable for our purposes (listed in additional file 9; data not shown).

In a subsequent set of experiments, the effects of GSAO vs. GSCA on specific signalling proteins in PWBC were investigated. The results are described and shown in additional text file and additional files 1 to 11 .

From this large data set we conclude that GSAO has rather pleiotropic effects on the cell signalling protein network of PWBC cultured in low serum conditions. Since it is very unlikely that a drug which affects numerous key signalling proteins would show the low toxicity profile seen in vivo [10], we modified the PWBC culture system.

\section{GSAO affects only a small subset of signalling proteins when PWBC are cultured in high serum}

Figure 2 shows a comparison of GSAO-induced effects on protein tyrosine phosphorylation of PWBC cultured in low serum $(0.5 \% \mathrm{FBS})$ versus $100 \%$ of donor serum. Culture of PWBC in 100\% donor serum reduces the overall level of tyrosine phosphorylation in non-treated and GSCA-treated cells. It also reduces the intensity of changes seen upon GSAO-treatment. Titration of GSAO showed that, in the presence of $100 \%$ donor serum, increased PWBC tyrosine phosphorylation is clearly observable with $15 \mu \mathrm{M}$ GSAO treatment of PWBC for $24 \mathrm{~h}$ (figure 2B).

Subsequent analyses focussed on GSAO effects on high serum-cultured PWBC with those phospho-epitope-specific antibodies, which had shown phosphorylation changes under low serum culture conditions. From these experiments, it is obvious that GSAO is affecting only a much smaller number of proteins in high serum culture.
For example, p90Rsk phosphorylation on Ser380 and phosphorylation of JNKs is at best barely observable in high serum culture (figure 3C and 3D; compare to additional files 3 and 5). However, phosphorylation of Erk2 on Thr202/Tyr204 is obvious after exposure of cells to 50 $\mu \mathrm{M}$ GSAO for $24 \mathrm{~h}$ (figure $3 \mathrm{~A}$ ). Because the trial will involve infusions of GSAO, more chronic exposure was studied. Extension of the GSAO exposure time on PWBC in high serum culture showed that $15 \mu \mathrm{M}$ GSAO induces Erk2 activation after $48 \mathrm{~h}$ and after $72 \mathrm{~h}$ a massive activation of Erk2 is seen (figure 3B).

The paxillin homologue Hic-5 (hydrogen oxide inducible clone- $\underline{5} ;[14]$ was one of the few other proteins which was clearly affected by GSAO under high serum culture conditions (figure 4). GSAO treatment induces a massive mobility shift of the Hic-5 band at a concentration of 15 $\mu \mathrm{M}$ compound. This upshift is probably a consequence of an altered phosphorylation state of Hic-5, however, other modifications of Hic-5 upon GSAO treatment of PWBC cannot be excluded. Phosphorylation of Hic -5 on Tyr38 and 60 has been previously reported $[15,16]$, but phospho-specific antibodies for these epitopes are unavailable. A slight shift in Hic-5 mobility was occasionally seen with as little as $5 \mu \mathrm{M}$ GSAO (figure $4 \mathrm{~B}$ ).
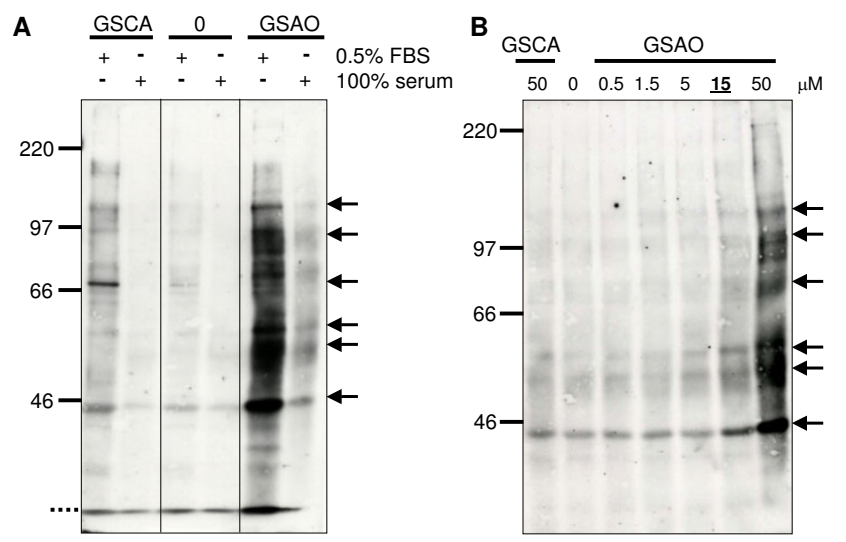

\section{Figure 2}

Effects of GSAO on protein tyrosine phosphorylation are also visible under high serum culture conditions. A PWBC were cultured in low serum $(0.5 \%$ FBS $)$ or $100 \%$ donor serum and treated with $50 \mu$ M GSAO or GSCA for 24 $\mathrm{h}$ where indicated. Overall, levels of protein tyrosine phosphorylation are lower in donor serum-cultured PWBC and the hyperphosphorylation of tyrosyl residues observed upon GSAO-treatment is less pronounced but still detectable. B GSAO dose-response of PWBC cultured in 100\% donor serum. PWBC were treated for $24 \mathrm{~h}$. GSAO-induced hyperphosphorylation on tyrosyl residues is clearly visible with 15 $\mu$ M GSAO. 
A

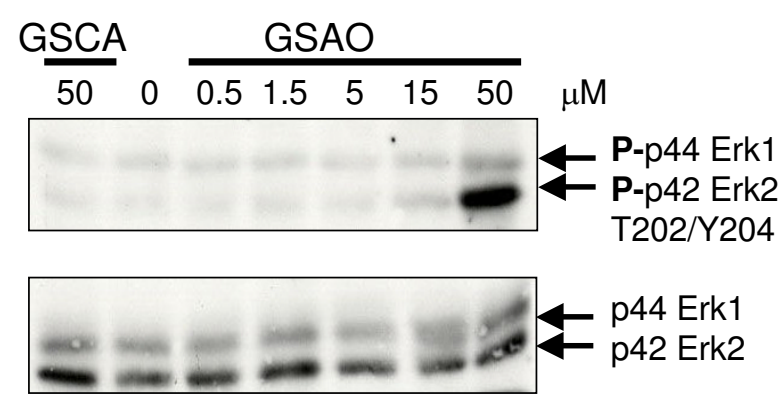

B

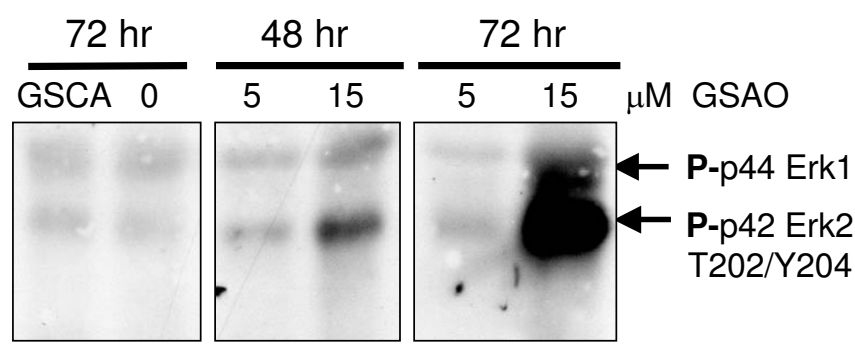

C

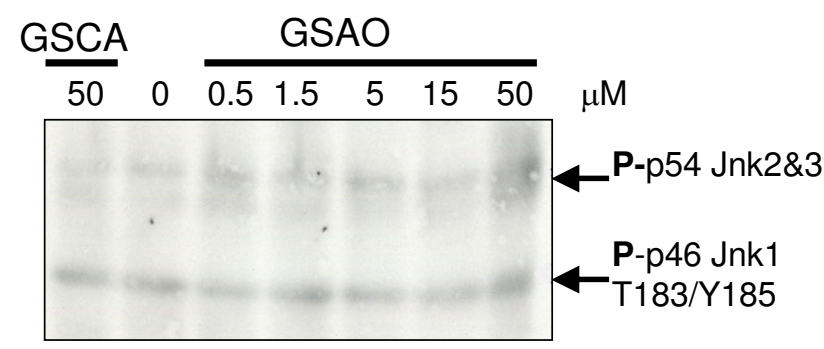

D

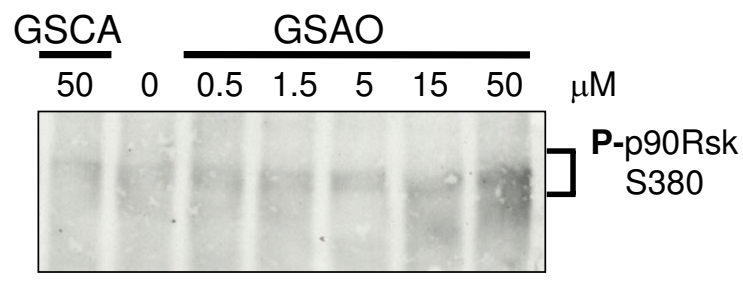

Figure 3

GSAO effects Erk, but not p90Rsk or Jnk phosphorylation in PWBC high serum culture. PWBC were cultured in $100 \%$ donor serum and treated with GSAO or GSCA for $24 \mathrm{~h}$ as indicated.A Phosphorylation of Erk2 on the Thr202/Tyr204 epitope is visible at $50 \mu \mathrm{M}$ GSAO (upper panel). Total Erk protein levels remain unchanged after GSAO treatment for $24 \mathrm{~h}$ (lower panel). B Phosphorylation of Ser380 on p90Rsk is marginally increased at best in high serum cultured PWBC. C Phosphorylation of the Thr 183/Tyr 185 epitope on Jnks is marginally increased at best in high serum cultured PWBC.

A third protein clearly affected by GSAO in high serum PWBC culture was a PRK1/PKNa antibody-immunoreactive band of $100 \mathrm{kDa}$ (figure 5). Protein kinase C-related kinases (PRKs, also designated PKNs) are known effectors of Rho GTPases and activated by fatty acids and lipids in vitro [17]. Phosphorylation of PRK1/PKN $\alpha$ Thr778 (corresponding to PRK2/PKN $\gamma$ Thr816) in the activation loop of the kinase leads to an increased catalytic activity of PRKs/PKNs. While PRK1/PKN $\alpha$ and PRK2/PKN $\gamma$ are ca. 120 and $140 \mathrm{kDa}$ in size, respectively, PKN $\beta$ has an apparent molecular weight of ca. $100 \mathrm{kDa}$. We were so far unable to determine whether the observed band is indeed PKN $\beta$, due to a lack of suitable specific reagents.

In summary, the screen with a large number of epitopespecific antibodies has resulted in the detection of three candidate surrogate markers for GSAO activity in PWBC under high serum conditions. To search for further potential markers, antibodies targeted against phosphorylated substrate epitopes or docking motifs of a specific kinase or kinase family were used on GSAO-, GSCA- or mocktreated PWBC lysates.

Detection of further proteins affected by GSAO in PWBC high serum culture using kinase substrate-specific antibodies

Phosphorylated substrate epitope-directed antibodies for the protein targets of the kinase families Akt, ATM/ATR, Cdk, MAPK, PKA, PKC and PKD, as well as a PDK-1 phospho-docking motif antibody (for details see additional file 9) were used to detect changes in kinase substrate proteins of PWBC cultured in high or low serum in the presence or absence of GSAO and GSCA. Selected results are shown in figures 6 and additional files 6 and 7. GSAOinduced protein phosphorylation changes were again, as expected, much more pronounced under low serum culture conditions and served as a useful control for the general functionality of the antibodies. GSAO-effects in high serum-cultured PWBC were observed with the Akt (figure 6), PKA (additional file 6) and PKD (additional file 7) 
A

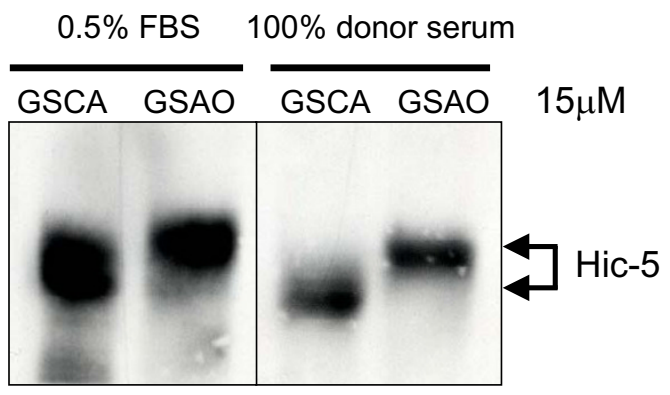

B

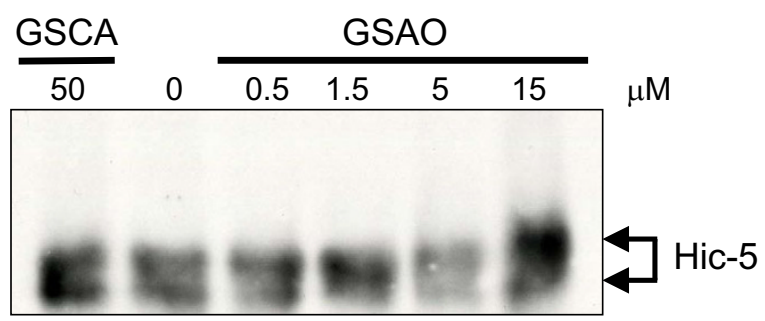

Figure 4

Prominent mobility change of Hic-5 in GSAOtreated PWBC in low and high serum culture. Cells were treated with GSCA or GSAO as indicated for $24 \mathrm{~h}$ and analysed for Hic-5 expression and mobility. A Comparisonof effects induced by $15 \mu \mathrm{M}$ GSAO under low and high serumculture conditions. In both cases a prominent band shift of Hic-5 isobserved. B GSAO dose - response of Hic-5 in PWBC culturedin 100\% donor serum. A partial mobility shift is observable at $5 \mu \mathrm{M}$ GSAO, but the band shift is more pronounced at I5 $\mu \mathrm{M}$ GSAO.

substrate-directed antibodies. These results show that a range of further proteins affected by GSAO-exposure of PWBC cultured in high serum remain to be identified.

\section{GSAO affects signalling proteins in HUVEC endothelial cells}

GSAO targets proliferating endothelial cells in vivo [10], therefore experiments were carried out with primary endothelial cells. Human umbilical vein endothelial cells (HUVEC) were treated with 15 or $50 \mu \mathrm{M}$ GSAO. Increased tyrosine phosphorylation was observed in the HUVEC upon $15 \mu \mathrm{M}$ treatment with GSAO (figure 7A). The PRK1/ PKNa Thr778 antibody-immunoreactive band of 100 $\mathrm{kDa}$ was not detectable in HUVEC upon treatment with GSAO and no changes in phosphorylation or protein expression of PRK1/PKN $\alpha$ were detectable (figure 7B). No induction of phosphorylation of Erk1/2 Thr202/Tyr204 was observed (figure 7B). However, GSAO treatment of HUVEC with $50 \mu \mathrm{M}$ GSAO clearly induces a mobility shift

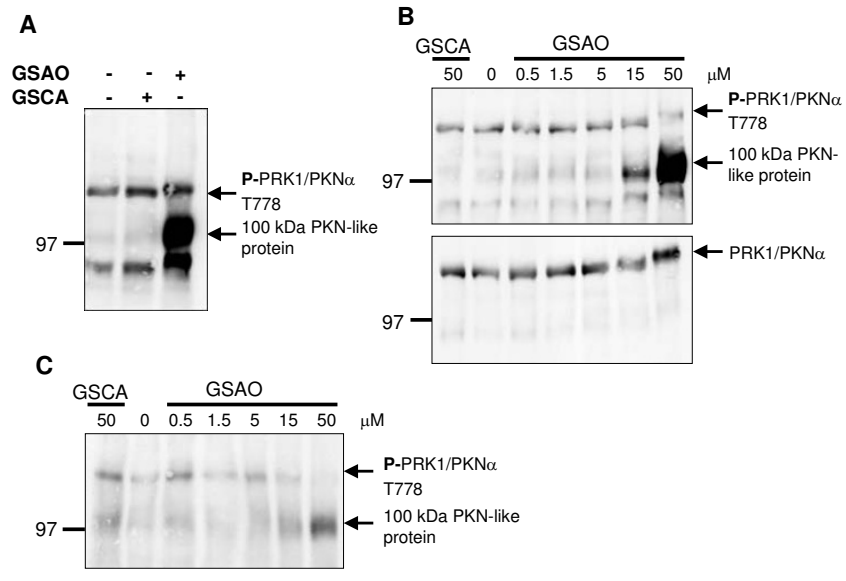

Figure 5

GSAO induces phosphorylation of a $100 \mathrm{kDa}$ protein which is recognised by an antibody directed against PRKI/PKN $\alpha$ pThr778. PWBC were treated with GSCA or GSAO where indicated for $24 \mathrm{~h}$ and proteins analysed with anti-PRKI/PKN $\alpha$ PThr778. A Effect of $50 \mu M$ GSAOvs. GSCA on a $100 \mathrm{kDa}$ protein recognised by anti-PRKI/PKN $\alpha$ PThr 778. A prominent hyperphosphorylation of the 100 $\mathrm{kDa}$ protein is visible, while a signal of ca. $120 \mathrm{kDa}$, the size expected for PRKI/PKN $\alpha$, remains unchanged. The $100 \mathrm{kDa}$ band may correspond to the PRKI/PKN $\alpha$ relative $P K N \beta$, but this can only be verified after the generation of PKN $\beta$-specific antibodies. B GSAO dose - response in low serum cultured PWBC. Hyperphosphorylation of the $100 \mathrm{kDa}$ band is visible from I $5 \mu$ M of GSAO with the PThr778-specific PRKI/PKN $\alpha$ antibody (upper panel) while total protein levels of PRKI/PKN $\alpha$ remain unchanged (lower panel). A mobility shift of PRKI/PKN $\alpha$ is also visible with this antibody at higher GSAO concentrations, possibly indicating a PRKI/PKN $\alpha$ phosphorylation at a site different from pThr778. C Effect of GSAO on the $100 \mathrm{kDa}$ band in high serum cultured PWBC. A moderate increase of phosphorylation at the PThr778related epitope is visible at $50 \mu \mathrm{M}$ GSAO.

of the Hic-5 band (see figure 7B) similar to that seen in PWBC cultured in high serum.

\section{Discussion}

STM drugs are playing an increasingly important role in cancer therapy. Many of these drugs appear to have low toxicity profiles, so biological responses are expected below drug concentrations, which lead to massive side effects. Based on the results from previous animal studies $[10,18]$, one can speculate that this may also be the case for novel anti-cancer compound GSAO. Surrogate markers that can be monitored to detect the biological activity of a STM drug in patients shortly after drug application may therefore be of great value for its optimal clinical development. 


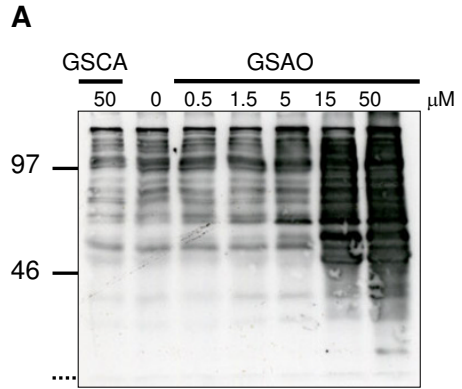

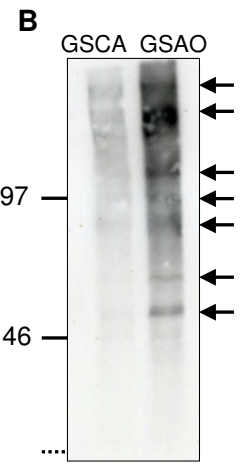

Figure 6

Detection of proteins hyperphosphorylated after treatment of PWBC with GSAO using an Akt substrate-directed antibody. Cells were incubated for 24 hwith GSAO or GSCA as indicated and analysed with an antibody made to recognise multiple Akt substrate proteins in their Akt-phosphorylated forms (R/K-x-R/K-x-x-pS/pT motif, Cell Signalling Technology 96II). A Massive effect of GSAO on phosphoproteins detected with the Akt substrate phospho-antibody using protein extracts from low serum cultured PWBC. The control compound GSCA does not detectably change the basal phospho-protein pattern. B Effects of $15 \mu \mathrm{M}$ GSAO on high serum culturedPWBC. Arrows indicate bands that are hyperphosphorylated inGSAO-treated cells.

GSAO is thought to primarily interfere with proliferating endothelial cells [10], hence disrupting the further growth of tumour vasculature. GSAO activity is therefore dependent on systemic distribution through the blood vessels and capillaries but probably does not need to penetrate deeply into tissues to exert key effects. As endothelial cells and PWBC are both key components of the blood vessel system and will be exposed to circulating GSAO to a similar degree, PWBC should be well suited for the analysis of surrogate markers for GSAO activity in vivo.

Our experiments clearly show that classical low serum culture, often used to reduce background signals prior to addition of a drug or factor, is not ideal to find potential markers for GSAO bioactivity in vivo. Furthermore, this study was only possible through the multitude of commercial signalling protein antibodies that have become available in the last few years. These antibodies, directed against specific phosphorylated or nonphosphorylated protein epitopes in signalling proteins, allow the rapid analysis of a significant part of the signalling phosphoproteome. In order to obtain reliable data with these antibodies, their specificity must be carefully evaluated. In our western blot experiments, we frequently found multiple
A

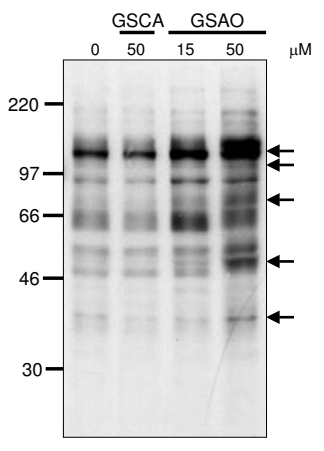

B

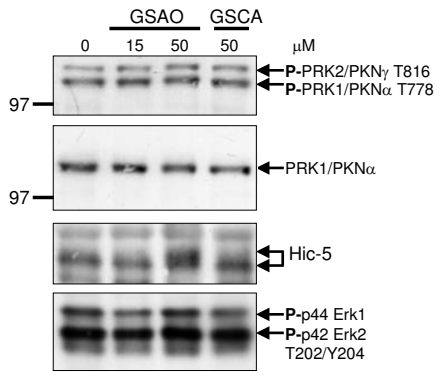

\section{Figure 7}

Effects of GSAO treatment on HUVEC. HUVEC wereincubated for $24 \mathrm{~h}$ with GSAO or GSCA as indicated with I5\% FBS. A Increased tyrosine phosphorylation is readily detectable at I5 $\mu$ M GSAO treatment. Some GSAO affected proteins are indicated witharrows. B Hyperphosphorylation of the $100 \mathrm{kDa}$ protein is undetectable in HUVEC, while a band of ca. $120 \mathrm{kDa}$, the size expected for PRKI/PKN $\alpha$, remains unchanged. A band shift of Hic-5 is detectable at 50 $\mu M$ GSAO treatment. Phosphorylation changes in Erk2 at Thr202/Tyr204 are not detectable.

bands resulting from a single antibody analysed. Signals appeared often at unexpected sizes, especially for polyclonal anti sera. Until now, high quality monoclonal antibodies are only available for a small number of phosphoepitopes. We feel that this would make, at present, results from high throughput (HTP) array-format assays or FACS-based HTP analyses like multiplex-bead assays difficult to properly evaluate.

Monitoring of drug effects on total protein expression levels, is simpler, but appears to be overall less informative as many phospho-changes are not mirrored by changes in overall protein expression. Nevertheless, it can give some initial leads and many good quality monoclonal antibodies are readily available. For optimal retrieval of information from western blot type assays it is necessary to perform non-automated visual re-inspections to detect all changes. The GSAO-induced Hic-5 mobility shift was found through this approach and would have been missed using a spot array or another technique not separating proteins by apparent molecular weight.

Hic-5 is an interesting candidate for further analyses of GSAO actions in cells. It is a member of the paxillin family $[19,20]$ and has been implicated in a variety of biological processes including those that regulate cell motility, invasion, survival and proliferation (summarised in [19]). 
Moreover, recent publications have highlighted additional roles for Hic-5, for example, the suppression of Lef/ Tcf-driven transcription during vertebrate development [21]. Hic-5 was also reported to undergo redox-sensitive nuclear-cytoplasmic shuttling $[22,23]$ and to interact with Traf4, which is implicated in the oxidative regulation of endothelial cell migration [24]. GSAO uptake and action has also been shown to depend on redox parameters like cellular glutathione levels [11], but it remains to be studied if GSAO directly influences theredox regulation of cells or cellular subcompartments, for example by interfering with redox-regulated phosphatases like PTP-PEST [24] or other signalling proteins.

The consistent activation of the MAP kinase Erk2 by GSAO under high and low serum conditions is also intriguing, since no activation of its regulator kinases MEK1/2 could be observed (data not shown). Apart from a direct effect on Erk-inactivating phosphatases, like MKPs, other more indirect changes in PWBC signalling may lead to Erk2 activation. Since the intracellular GSAO uptake is very slow and effects are usually seen after many hours, a plethora of possible indirect mechanisms, including changes in the transcription of certain genes may underlie the observed Erk2 activity change. Erk2 activation is thought to trigger its dimerisation and nuclear translocation [25], possibly leading to the phosphorylation of nuclear substrate proteins. Analyses of selected Erk substrates like p90Rsk, and Elk-1 with phospho-epitope specific antibodies (results summarised in additional file 9) did not pinpoint an affected Erk2 substrate so far and an initial screen for substrates of proline-directed kinases (like Erks and Cdks) with an antibody which recognises an xxx-pThr-Pro-xxx motif did also not detect candidate substrates in GSAO-treated PWBC culture in high serum (not shown). Since more than 70 Erk substrates have been reported so far, a large number of potential substrates remain to be analysed. Beyond this, experiments with substrate epitope-directed phospho-antibodies specific for targets of other kinases clearly indicate that additional GSAO-affected proteins, which could be useful bioactivity markers, remain to be discovered.

Changes in one of the three candidate surrogate markers for GSAO activity found in PWBC is detectable in endothelial cells, but additional changes in tyrosine phosphorylation were observed in a multitude of proteins at $15 \mu \mathrm{M}$ GSAO treatment in HUVEC. This suggests that overlapping, but distinct groups of signalling pathways are affected by GSAO in the different cell types. Further studies into the biological mechanisms of GSAO interference with endothelial cell proliferation are needed to elucidate the in vivo activity of GSAO in this cell type.

\section{Conclusion}

This study has shown that GSAO effects on the PWBC phosphoprotein signalling network are quite selective, provided the appropriate experimental conditions are used. The results also give suggestions for the development of new reagents, which may be useful to support future clinical studies and to possibly gain further insight into the cellular targets of GSAO. Analyses with substrate epitope-specific antibodies show that further GSAOaffected proteins remain to be identified. Variation in phosphorylation patterns between PWBC and HUVEC suggest distinct pathway patterns become activated by GSAO in different cell types. As PWBC are easily obtainable they are more probably suitable for measuring surrogate markers in future clinical studies, but endothelial cells are clearly also an important cell type that needs to be analysed further with molecular and functional assays.

\section{Competing interests}

All authors declare not to have any competing interests. Cancer Research UK, which funds the work of VAC, SMF and $\mathrm{ALH}$, is a sponsor and organiser of the planned GSAO clinical trial.

\section{Authors' contributions}

VAC performed most of the experiments and participated in the drafting of the manuscript. PJH provided the GSCA and GSAO and critically commented on the drafted manuscript. ALH participated in the design of some experiments and critically commented on the drafted manuscript. SMF designed the study, drafted the manuscript and performed some initial experiments.

\section{Additional material}

\section{Additional File 1}

GSAO and phenylarsine oxide (PAO) induce similar patterns of tyrosine phosphorylation in peripheral white blood cells (PWBC). Freshly isolated PWBC were equilibrated in medium with 0.5\% FBS for $2 h$ and then treated with the indicated concentrations of PAO or GSAO. PWBC were subsequently lysed and proteins analysed for protein tyrosine phosphorylation by western blot. Uptake of the hydrophilic GSAO into cells is, as expected, very slow. Treatment of PWBC with $50 \mu \mathrm{M} G S A O$ for $8 \mathrm{~h}$ results in protein tyrosyl-hyperphosphorylation similar to that seen upon incubation with $1 \mu \mathrm{M}$ PAO for $1 \mathrm{~h}$. Hyperphosphorylated protein bands are indicated by arrows.

Click here for file

[http://www.biomedcentral.com/content/supplementary/14712407-6-155-S1.PPT] 


\section{Additional File 2}

Dose-dependent increase of PWBC protein threonine phosphorylation induced by GSAO. PWBC were treated with GSAO or GSCA where indicated for $24 \mathrm{~h}$ and threonine phosphorylation detected by western blot. A $50 \mu \mathrm{M}$ GSCA does not alter the protein pThr levels of PBWC detectably. $B$ Changes in protein pThr patterns are apparent with as little as $1.5 \mu \mathrm{M}$ GSAO. GSAO-affected proteins are indicated with arrows.

Click here for file

[http://www.biomedcentral.com/content/supplementary/14712407-6-155-S2.PPT]

\section{Additional File 3}

GSAO induces phosphorylation of c-Jun N-terminal kinases (JNKs) at the kinase activity-regulating epitope (Thr 183/Tyr 185) in PWBC. PWBC were treated with GSAO or GSCA, then lysed and analysed by western blot for JNK expression or phosphorylation. The phospho-specific antibody detects JNK protein only effectively when doubly phosphorylated on Thr183 and Tyr185. A Cells were treated with $50 \mu \mathrm{M}$ GSAO or GSCA for $24 \mathrm{~h}$ where indicated and analysed for phospho-JNK (upper panel) or total JNK (lower panel) B Cells were treated with different concentrations of GSAO for $24 \mathrm{~h}$ as indicated and analysed for phospho-JNK.

Click here for file

[http://www.biomedcentral.com/content/supplementary/14712407-6-155-S3.ppt]

\section{Additional File 4}

Effects of GSAO on the MAP kinase cascade. Cells were treated with GSAO or GSCA as indicated for $24 \mathrm{~h}$ and lysates analysed for the phosphorylation of Erk1/Erk2 (pThr202/pTyr204) or c-Raf-1 (pTyr340/ pTyr341; pSer259). Phosphorylation of Erk1/Erk2 at the epitope Thr202/ Tyr204 correlates closely with Erk kinase activation. The phospho-epitopes analysed for $c$-Raf-1 also contribute to the regulation of c-Raf-1 kinase activity, but numerous other regulatory sites have been described in $c$-Raf1. A Effect of GSAO treatment on the phosphorylation of Erk1/Erk2 (pThr202/pTyr204) detected by a polyclonal antibody (9101, Cell Signalling Technology). Apart from the major Erk1/2 phospho-bands, several additional phospho-bands of different sizes, some possibly representing other Erk family members, are visible. B Effect of GSAO treatment on the phosphorylation of Erk1/Erk2 (pThr 202/pTyr204) detected by a monoclonal antibody (M8159, Sigma). C Effect of GSAO treatment on the phosphorylation of c-Raf-1 at Tyr340/Tyr341. Phosphorylation of this epitope can contribute to the activation of Raf kinase. D Effect of GSAO treatment on the phosphorylation of c-Raf-1 at Ser259. Dephosphorylation of this epitope is thought to be important for activation of the kinase. The c-Raf-1 band shift observed with 15 and $50 \mu \mathrm{M}$ GSAO, probably resulting from phosphorylation events at other $c$-Raf-1 epitopes, makes it difficult to determine if a partial dephosphorylation of pSer259 occurs at these GSAO concentrations.

Click here for file

[http://www.biomedcentral.com/content/supplementary/14712407-6-155-S4.ppt]

\section{Additional File 5}

Effects of GSAO on the phosphorylation of p90Rsk at Ser380 in $P W B C$ obtained from different volunteers. $P W B C$ were treated with GSAO or GSCA as indicated for $24 \mathrm{~h}$ and lysates analysed for the phosphorylation of Ser380 in p90Rsk. Cells from three different donors were used. The male donor was analysed on two different occasions. Increased Ser380 phosphorylation is clearly visible at $5 \mu \mathrm{M}$ GSAO in all cases. With $50 \mu \mathrm{M}$ GSAO a major mobility shift of p90Rsk (pSer380) is visible. This could indicate the occurrence of additional phosphorylation events.

Click here for file

[http://www.biomedcentral.com/content/supplementary/14712407-6-155-S5.ppt]

\section{Additional File 6}

Detection of proteins hyperphosphorylated after treatment of PWBC with GSAO using a PKA substrate-directed antibody. Cells were incubated for $24 \mathrm{~h}$ with GSAO or GSCA as indicated and analysed with an antibody made to recognise multiple PKA substrate proteins in their PKAphosphorylated forms (R-R-x-pS or R-x-x-pT motif, Cell Signalling Technology 9621). A Strong effect of GSAO on phosphoproteins detected with the PKC substrate phospho-antibody using protein extracts from low serum cultured PWBC. The control compound GSCA does not detectably change the basal phospho-protein pattern. B Effects of $15 \mu \mathrm{M}$ GSAO on high serum cultured PWBC. Arrows indicate bands that are slightly hyperphosphorylated in GSAO-treated cells.

Click here for file

[http://www.biomedcentral.com/content/supplementary/14712407-6-155-S6.ppt]

\section{Additional File 7}

Detection of proteins hyper-phosphorylated after treatment of PWBC with GSAO using a PKD substrate-directed antibody. Cells were incubated for $24 \mathrm{~h}$ with GSAO or GSCA as indicated and analysed with an antibody made to recognise multiple PKD substrate proteins in their PKDphosphorylated forms ( $L-x-R-x-x-p T / p S$ motif, Cell Signalling Technology 4381) A Effect of GSAO on phosphoproteins detected with the PKC substrate phospho-antibody using protein extracts from low serum cultured $P W B C$. The control compound GSCA does not detectably change the basal phospho-protein pattern. B Effects of $15 \mu \mathrm{M} \mathrm{GSAO}$ on high serum cultured PWBC. Arrows indicate three bands that are clearly hyperphosphorylated in GSAO-treated cells.

Click here for file

[http://www.biomedcentral.com/content/supplementary/14712407-6-155-S7.ppt]

\section{Additional File 8}

Compiled results of the Powerblot ${ }^{\mathrm{TM}}$ comparison of PWBC cultured in low serum and treated with $50 \mu \mathrm{M}$ GSAO or GSCA.

Click here for file

[http://www.biomedcentral.com/content/supplementary/14712407-6-155-S8.xls]

\section{Additional File 9}

Antibodies used for analysis of GSAO effects on PWBC. Click here for file

[http://www.biomedcentral.com/content/supplementary/14712407-6-155-S9.xls] 


\section{Additional File 10}

Summary of GSAO-induced protein expression changes in PWBC cultured with low serum medium. Of 240 individually selected signalling proteins, which were analysed by the commercially available Powerblot ${ }^{\mathrm{Tm}}$, the 24 proteins listed here showed an apparent change in protein expression considered to be highly significant according to the company. Proteins in blue color are upregulated, proteins in black downregulated with GSAO vs. GSCA. From this list it is obvious, that kinases and PTPases and their targets make up the majority of proteins affected in their expression level by GSAO. At least two of the signals (LAT, VASP) are, however, clearly questionable, as the detected band size in the sample varies greatly from the expected protein size. In other cases, for example Hic-5, an expression change is detected by the automated system. Upon visual inspection this supposed expression change results from a mobility shift of Hic 5. A full list of all proteins analysed and changes detected are compiled in 3.

Click here for file

[http://www.biomedcentral.com/content/supplementary/1471-

2407-6-155-S10.ppt]

\section{Additional File 11}

Pleiotropic effects on PWBC in low serum conditions.

Click here for file

[http://www.biomedcentral.com/content/supplementary/14712407-6-155-S11.doc]

\section{Acknowledgements}

We are grateful to the volunteers for donating blood and to Cancer Research UK for funding our work.

\section{References}

I. Alonso A, Sasin J, Bottini N, Friedberg I, Osterman A, Godzik A, Hunter T, Dixon J, Mustelin T: Protein tyrosine phosphatases in the human genome. Cell 2004, II 7(6):699-7II.

2. Andersen JN, Jansen PG, Echwald SM, Mortensen OH, Fukada T, Del Vecchio R, Tonks NK, Moller NP: A genomic perspective on protein tyrosine phosphatases: gene structure, pseudogenes, and genetic disease linkage. Faseb J 2004, I 8(I):8-30.

3. Wang Z, Shen D, Parsons DW, Bardelli A, Sager J, Szabo S, Ptak J, Silliman N, Peters BA, van der Heijden MS, Parmigiani G, Yan H, Wang TL, Riggins G, Powell SM, Willson JK, Markowitz S, Kinzler KW, Vogelstein B, Velculescu VE: Mutational analysis of the tyrosine phosphatome in colorectal cancers. Science 2004, 304(5674): II64-II66.

4. Ducruet AP, Vogt A, Wipf P, Lazo JS: Dual specificity protein phosphatases: therapeutic targets for cancer and Alzheimer's disease. Annu Rev Pharmacol Toxicol 2005, 45:725-750.

5. Gasparotto D, Maestro R, Piccinin S, Vukosavljevic T, Barzan L, Sulfaro S, Boiocchi M: Overexpression of CDC25A and CDC25B in head and neck cancers. Cancer Res 1997, 57( 12 2):2366-2368.

6. Guo J, Kleeff J, Li J, Ding J, Hammer J, Zhao Y, Giese T, Korc M, Buchler MW, Friess $\mathrm{H}$ : Expression and functional significance of CDC25B in human pancreatic ductal adenocarcinoma. Oncogene 2004, 23(I):7I-8I.

7. Pestell KE, Ducruet AP, Wipf P, Lazo JS: Small molecule inhibitors of dual specificity protein phosphatases. Oncogene 2000, 19(56):6607-66I2.

8. Cristofanilli M, Charnsangavej C, Hortobagyi GN: Angiogenesis modulation in cancer research: novel clinical approaches. Nat Rev Drug Discov 2002, I(6):4I5-426.

9. Donoghue N, Yam PT, Jiang XM, Hogg PJ: Presence of closely spaced protein thiols on the surface of mammalian cells. Protein Sci 2000, 9( I 2):2436-2445.

10. Don AS, Kisker O, Dilda P, Donoghue N, Zhao X, Decollogne S, Creighton B, Flynn E, Folkman J, Hogg PJ: A peptide trivalent arsenical inhibits tumor angiogenesis by perturbing mito- chondrial function in angiogenic endothelial cells. Cancer Cell 2003, 3(5):497-509.

II. Dilda PJ, Don AS, Tanabe KM, Higgins VJ, Allen JD, Dawes IW, Hogg PJ: Mechanism of selectivity of an angiogenesis inhibitor from screening a genome-wide set of Saccharomyces cerevisiae deletion strains. J Natl Cancer Inst 2005, 97(20): 1539- 1547

12. Defilippi P, Retta SF, Olivo C, Palmieri M, Venturino M, Silengo L, Tarone G: p I 25FAK tyrosine phosphorylation and focal adhesion assembly: studies with phosphotyrosine phosphatase inhibitors. Exp Cell Res 1995, 22 I (I): | 14I-I52.

13. Oetken C, von Willebrand M, Autero M, Ruutu T, Andersson LC, Mustelin T: Phenylarsine oxide augments tyrosine phosphorylation in hematopoietic cells. Eur J Haematol 1992, 49(4):208-2। 4 .

14. Shibanuma M, Mashimo J, Kuroki T, Nose K: Characterization of the TGF beta I-inducible hic-5 gene that encodes a putative novel zinc finger protein and its possible involvement in cellular senescence. J Biol Chem 1994, 269(43):26767-26774.

15. Hetey SE, Lalonde DP, Turner CE: Tyrosine-phosphorylated Hic5 inhibits epidermal growth factor-induced lamellipodia formation. Exp Cell Res 2005, 3 I I(I): I47-I56.

16. Ishino M, Aoto H, Sasaski H, Suzuki R, Sasaki T: Phosphorylation of Hic-5 at tyrosine 60 by CAKbeta and Fyn. FEBS Lett 2000, 474(2-3): $179-183$.

17. Mukai $H$ : The structure and function of $P K N$, a protein kinase having a catalytic domain homologous to that of PKC. J Biochem (Tokyo) 2003, 133(1): 17-27.

18. Dilda PJ, Decollogne S, Rossiter-Thornton M, Hogg PJ: Para to ortho repositioning of the arsenical moiety of the angiogenesis inhibitor 4-(N-(S-glutathionylacetyl)amino)phenylarsenoxide results in a markedly increased cellular accumulation and antiproliferative activity. Cancer Res 2005, 65(24): $11729-11734$

19. Brown MC, Turner CE: Paxillin: adapting to change. Physiol Rev 2004, 84(4): I3I5-1339.

20. Turner CE: Paxillin and focal adhesion signalling. Nat Cell Biol 2000, 2(I 2):E23 I-6.

21. Ghogomu SM, Vanvenrooy S, Ritthaler M, Wedlich D, Gradl D: Hic5 , a novel repressor of Lef/Tcf driven transcription. J Biol Chem 2005.

22. Shibanuma M, Kim-Kaneyama JR, Ishino K, Sakamoto N, Hishiki T, Yamaguchi K, Mori K, Mashimo J, Nose K: Hic-5 communicates between focal adhesions and the nucleus through oxidantsensitive nuclear export signal. Mol Biol Cell 2003, 14(3): |158-117|.

23. Shibanuma M, Mori K, Kim-Kaneyama JR, Nose K: Involvement of FAK and PTP-PEST in the regulation of redox-sensitive nuclear-cytoplasmic shuttling of a LIM protein, Hic-5. Antioxid Redox Signal 2005, 7(3-4):335-347.

24. Wu RF, Xu YC, Ma Z, Nwariaku FE, Sarosi GAJ, Terada LS: Subcellular targeting of oxidants during endothelial cell migration. J Cell Biol 2005, I 7 I(5):893-904.

25. Cobb MH, Goldsmith Ej: Dimerization in MAP-kinase signaling. Trends Biochem Sci 2000, 25(I):7-9.

\section{Pre-publication history}

The pre-publication history for this paper can be accessed here:

http://www.biomedcentral.com/1471-2407/6/155/pre pub 